\title{
Superdiffusive trajectories in Brownian motion
}

\author{
Jérôme Duplat \\ Service des Basses Températures, UMR-E 9004, CEA/UJF-Grenoble 1, INAC, Grenoble F-38054, France
}

Simon Kheifets, Tongcang Li, and Mark G. Raizen

Center for Nonlinear Dynamics and Department of Physics, University of Texas at Austin, Austin, Texas 78712, USA

\author{
Emmanuel Villermaux* \\ Institut de Recherche sur les Phénomènes Hors Equilibre, Aix-Marseille Université, 13384 Marseille Cedex 13, France
}

(Received 26 November 2012; published 15 February 2013)

\begin{abstract}
The Brownian motion of a microscopic particle in a fluid is one of the cornerstones of statistical physics and the paradigm of a random process. One of the most powerful tools to quantify it was provided by Langevin, who explicitly accounted for a short-time correlated "thermal" force. The Langevin picture predicts ballistic motion, $\left\langle x^{2}\right\rangle \sim t^{2}$ at short-time scales, and diffusive motion $\left\langle x^{2}\right\rangle \sim t$ at long-time scales, where $x$ is the displacement of the particle during time $t$, and the average is taken over the thermal distribution of initial conditions. The Langevin equation also predicts a superdiffusive regime, where $\left\langle x^{2}\right\rangle \sim t^{3}$, under the condition that initial velocity is fixed rather than distributed thermally. We analyze the motion of an optically trapped particle in air and indeed find $t^{3}$ dispersion. This observation is a direct proof of the existence of the random, rapidly varying force imagined by Langevin.
\end{abstract}

DOI: 10.1103/PhysRevE.87.020105

PACS number(s): 05.40.-a, 51.20.+d

Our modern understanding of Brownian motion dates back to the turn of the 20th Century, when Einstein [1] and Smoluchowski [2] synthesized equilibrium thermodynamics, viscous fluid mechanics, and the macroscopic diffusion laws established by Fourier [3]. An alternative vision was shortly after developed by Langevin [4], who introduced a stochastic differential equation for the motion of a particle in a thermal bath, with an explicit rapidly varying force $f(t)$ constantly exerted on the particle by the fluid molecules of the bath.

The Langevin equation governs the velocity $v=\dot{x}$ of a free particle in a viscous fluid, in the absence of hydrodynamic memory effects:

$$
\begin{aligned}
\dot{v} & =-v / \tau+f / m \\
\left\langle f(t) f\left(t^{\prime}\right)\right\rangle & =m^{2} \epsilon \delta\left(t-t^{\prime}\right),
\end{aligned}
$$

where $m$ is the particle mass, and $\tau=m / 6 \pi \eta a$ is the viscous relaxation time of the spherical particle of radius $a$ in a fluid with viscosity $\eta$. The force $f$ is "delta-correlated," meaning that its fluctuations are present down to arbitrarily short-time scales. This is equivalent to having a flat spectrum up to arbitrarily high frequencies; hence, it is referred to as white noise. The variance of the particle velocity is $\left\langle v^{2}\right\rangle_{\mathrm{eq}}=\epsilon \tau$ at equilibrium, and thanks to the equipartition theorem is such that $\left\langle v^{2}\right\rangle_{\text {eq }}=k_{B} T / m$, thus, relating temperature $T$ and damping rate $1 / \tau$ with the magnitude of the random force, a manifestation of the fluctuation-dissipation theorem.

Langevin did not exhaust all the riches of his model but used it to compute the ensemble average squared position of the particle in the long-time limit $(t \gg \tau)$ and found, for particles all starting at $x_{0}=0$, that

$$
\left\langle x^{2} \mid x_{0}=0\right\rangle=2 \epsilon \tau^{2} t,
$$

\footnotetext{
*villermaux@irphe.univ-mrs.fr
}

hence bridging Brownian motion, random walks, and diffusion, a view soon quantified experimentally by Perrin [5].

In a different context, Taylor [6] (and Fürth [7]) devised a similar model and reached the same conclusion regarding the existence of a diffusive régime [Eq. (2)] but also noted the existence of ballistic behavior, $\left\langle x^{2}\right\rangle=\epsilon \tau t^{2}$, in the shorttime limit $t \ll \tau$. This result, however, still depends on an average being performed on an initial condition of particles with distributed velocities. We come back below to that crucial point, not singled-out as such by either Taylor or Langevin.

Consider a particle released at $t=0$ in a thermalized medium (i.e., a bath of uniform temperature $T$ ), with constrained initial velocity $v_{0}$ (whether it is equal to zero or not) and position $x_{0}=0$. Solving the Langevin model [Eq. (1)] for the displacement $x(t)$ gives a mean

$$
\left\langle x \mid x_{0}=0, v_{0}\right\rangle=v_{0} \tau\left(1-e^{-t / \tau}\right),
$$

where $\left\langle\cdot \mid x_{0}, v_{0}\right\rangle$ denotes an average over many realizations of the thermal force, with fixed initial conditions (as opposed to an average over the thermal distribution of initial conditions). The mean trajectory is the same as it would be in the absence of the random force. However, solving Eq. (1) for the variance of the position gives

$$
\left\langle x^{2} \mid x_{0}\right\rangle=\left\langle x \mid x_{0}, v_{0}\right\rangle^{2}+\epsilon \tau^{2}\left(2 t-3 \tau+4 \tau e^{-t / \tau}-\tau e^{-2 t / \tau}\right) .
$$

The second term describes the broadening of the distribution of the particle's position $p_{x_{0}, v_{0}}(x, t)$ caused by the random force [8],

$$
\begin{aligned}
p_{x_{0}, v_{0}}(x, t) & =\exp \left[-\frac{\left(x-\left\langle x \mid x_{0}, v_{0}\right\rangle\right)^{2}}{2 \sigma_{x_{0}, v_{0}}^{2}}\right] / \sqrt{4 \pi \sigma_{x_{0}, v_{0}}^{2}} \\
\sigma_{x_{0}, v_{0}}^{2} & =\left\langle x^{2} \mid x_{0}, v_{0}\right\rangle-\left\langle x \mid x_{0}, v_{0}\right\rangle^{2}
\end{aligned}
$$


and the transition between short-time diffusion determined by the initial conditions and long-time diffusion, which is independent of the initial conditions.

Equation (4) has been known since Ornstein and Uhlenbeck [9-12], but it is typically averaged over the thermal distribution of the velocity since, until recently, it has not been possible to measure the instantaneous initial velocity of a Brownian trajectory. The short-time behavior $(t \ll \tau)$ of $\left\langle x \mid v_{0}\right\rangle$ is $v_{0} t$. An average over the thermal distribution of initial velocity (or equivalently setting $\left\langle v_{0}^{2}\right\rangle=\epsilon \tau$ ) gives Taylor's ballistic regime $\sigma^{2}=\epsilon \tau t^{2}$. If, however $v_{0}$ is not distributed, the first term in Eq. (4) vanishes. Expanding the second term in powers of $t$ reveals that the lowest nonvanishing term is

$$
\left\langle x^{2} \mid x_{0}=0, v_{0}=0\right\rangle=\frac{2}{3} \epsilon t^{3},
$$

which is very different from Eq. (2), exhibiting a superdiffusive (and even super-ballistic) regime, shown here as a special property of the Langevin model. This regime can also be observed despite a nonzero initial dispersion of the velocity. The effect of the random force is to make the velocity variance grow like $\left\langle v^{2}\right\rangle=\left\langle v_{0}^{2}\right\rangle+\epsilon t$ when $t<\tau$, until it reaches the thermalized asymptotic value $\epsilon \tau$. Thus, provided $\left\langle v_{0}^{2}\right\rangle \ll \epsilon \tau$, superdiffusion exists.

The source of the $t^{3}$ dependence in Eq. (6) is not immediately obvious: for zero, or small particle velocity, the damping term $v / \tau$ in Eq. (1) is negligible in front of the random force. The particle dispersion acceleration is thus such that $\ddot{x}=f / m$ to leading order. Integrating twice, squaring, then averaging provide the squared displacement

$$
\sigma_{x_{0}, v_{0}}^{2}=\frac{4}{m^{2}} \int_{0}^{t} d t_{1} \int_{0}^{t_{1}} d t_{2} \int_{0}^{t_{2}} d t_{3} \int_{0}^{t_{3}} d t_{4}\left\langle f\left(t_{2}\right) f\left(t_{4}\right)\right\rangle,
$$

which, due to the $\delta$ correlation of $f$, gives Eq. (6).

The Langevin dynamics in Eq. (1) correctly predicts the diffusive motion of Brownian particles immersed in a thermal bath at all time scales. In the case of a thermal distribution of initial conditions, the long- and short-time predictions have been confirmed experimentally [5,13-15]. In order to observe super-diffusion in standard Brownian motion, it is necessary to perform conditional statistics on Brownian trajectories with fixed (or narrowly distributed) initial velocity, so that dispersion statistics can be calculated for sets of trajectories with zero (or very small) relative initial velocity. The precise measurement of the instantaneous velocity of a Brownian particle is notoriously difficult due to its small magnitude and rapid fluctuation.

We have analyzed the trajectory of a microsphere trapped by an optical tweezer in air at two different pressures. In the experiments by $\mathrm{Li}$ et al. [13], a 3- $\mu$ m-diameter silica bead is trapped by a dual beam counter-propagating optical trap about some equilibrium position $(x=0)$. The frequency of the trap, $\omega_{0}=20 \times 10^{3} \mathrm{rad} / \mathrm{s}$, is such that $\tau=2.92 / \omega_{0}$ at low pressure (1/36 of atmosphere) and $\tau=0.92 / \omega_{0}$ at atmospheric pressure. Since $\omega_{0} \tau$ is of order unity, the fully diffusive regime obscured by oscillation within the trap; however, the acquisition frequency of the particle position is 2 $\mathrm{MHz}$ so that the short-time behavior is well resolved.

The equation of motion of the bead requires modification of Eq. (1) due to the presence of the trapping potential and
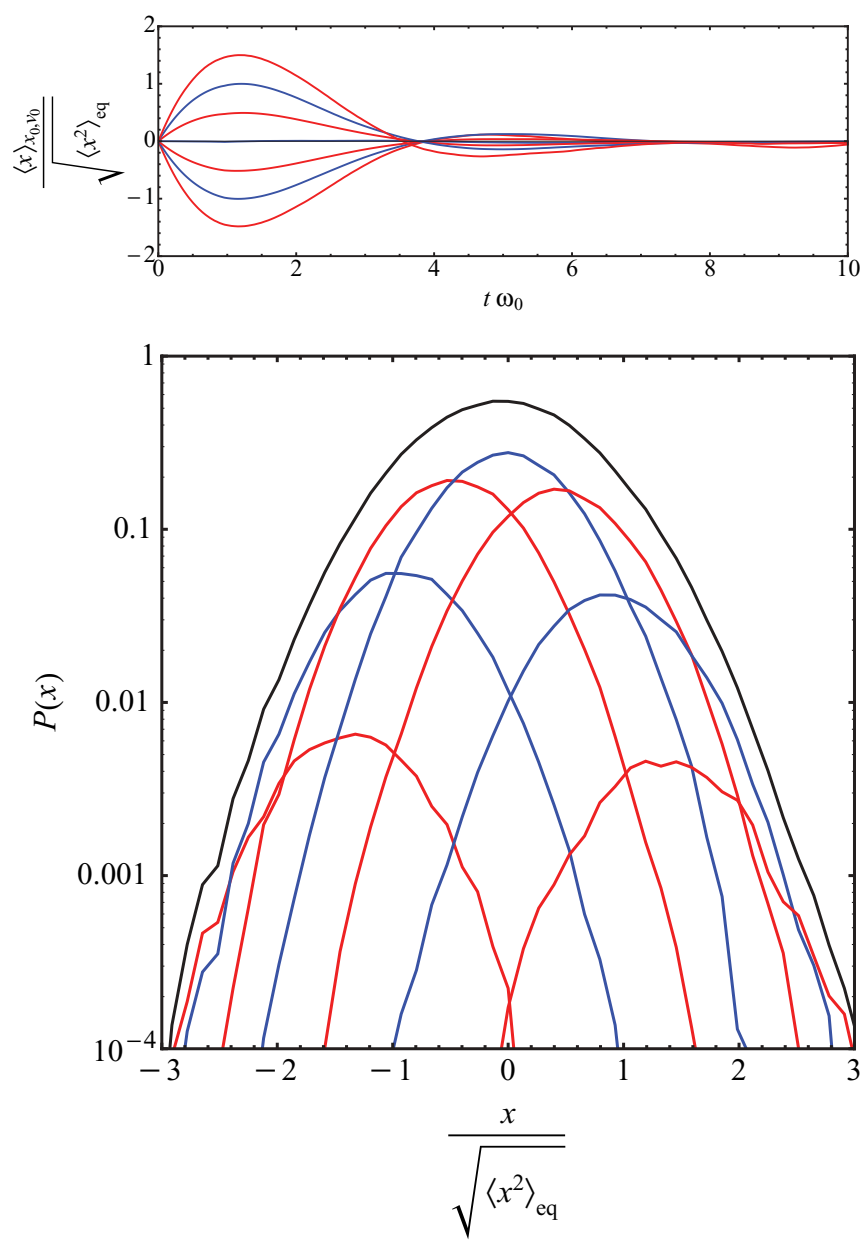

FIG. 1. (Color online) Top: average position of subsets of particles having a given initial velocity $v_{0}$. Bottom: distribution of the position of particles at $\omega t=0.97$ for subsets of particles having a given initial position $x_{0}=0$ and its decomposition in subset of fixed initial velocity $v_{0}$ (with $v_{0} / \sqrt{\left\langle v^{2}\right\rangle_{\mathrm{eq}}}=-3,-2,-1,0,1,2,3$ ). Data from the high pressure experiment of Ref. [13].

may be written as [11]

$$
\begin{aligned}
& \dot{x}=v \\
& \dot{v}=-\omega_{0}^{2} x-v / \tau+f / m \\
& \left\langle f(t) f\left(t^{\prime}\right)\right\rangle=m^{2} \epsilon \delta\left(t-t^{\prime}\right)
\end{aligned}
$$

where $\omega_{0}$ is the resonant frequency of the harmonic trap. For simplicity, we restrict ourselves to the case where $x_{0}=0$. The latter has little influence on the dispersion properties for times shorter than $1 / \omega_{0}$, and the super-dispersion regime can clearly be observed at short-time scales. On the contrary, at very large times, the particle becomes thermally equilibrated and the particle position is, accordingly with Eq. (5), Gaussianly distributed in the trap (Fig. 1), with a variance $\left\langle x^{2}\right\rangle_{\mathrm{eq}}=\epsilon \tau / 2 \omega_{0}^{2}$ determined by equipartition [8].

For intermediate times, the equation of motion of individual particles can be integrated, and the dispersion evolution is obtained. Introducing the corrected frequency $\omega_{1}=\sqrt{\omega_{0}^{2}-1 / 4 \tau^{2}}$ of the damped oscillator, one has, for particles all starting at $x_{0}=0$

$$
\frac{\sigma_{x_{0}}^{2}}{\left\langle x^{2}\right\rangle_{\mathrm{eq}}}=1-\exp \left(-\frac{t}{\tau}\right)\left[\cos \left(\omega_{1} t\right)+\frac{\sin \left(\omega_{1} t\right)}{2 \omega_{1} \tau}\right]^{2} \text {. }
$$


The total dispersion of the position $x$ is due to the contribution of two independent effects, namely the distribution of the random force and of the initial velocity (as discussed above). One has

$$
\sigma_{x_{0}}^{2}=\sigma_{x_{0}, v_{0}}^{2}+\sigma_{x_{0}, f}^{2},
$$

where subscripts refer to quantities maintained fixed: the first term denotes the dispersion due to random force (i.e., for a fixed initial velocity $v_{0}$ ), and the second denotes the effect of initial velocity dispersion (i.e., for a given realization $f(t)$ of the random force). The dispersion due to random force is

$$
\frac{\sigma_{x_{0}, v_{0}}^{2}}{\left\langle x^{2}\right\rangle_{\mathrm{eq}}}=1-\exp \left(-\frac{t}{\tau}\right)\left[\frac{\sin ^{2}\left(\omega_{1} t\right)}{2 \omega_{1}^{2} \tau^{2}}+\frac{\sin \left(2 \omega_{1} t\right)}{2 \omega_{1} \tau}+1\right] \text {. }
$$

The data were originally used to observe the ballistic motion of the particle, averaged blindly over all initial velocities [13]. Here, however, we separate trajectories into subsets with narrow relative velocity distributions and consider only the case where the initial position is close to zero. We calculate the dispersion $\sigma_{x_{0}, v_{0}}^{2}$ for each subset separately. In this way, the distribution of initial conditions for each subset is much smaller than the thermal distribution, and the dispersion of trajectories in each subset is caused only by the contribution from the random force. We find, as predicted, the characteristic $t^{3}$ super diffusive regime, as shown in Fig. 2. The magnitude of the relative dispersion corresponds to very small absolute

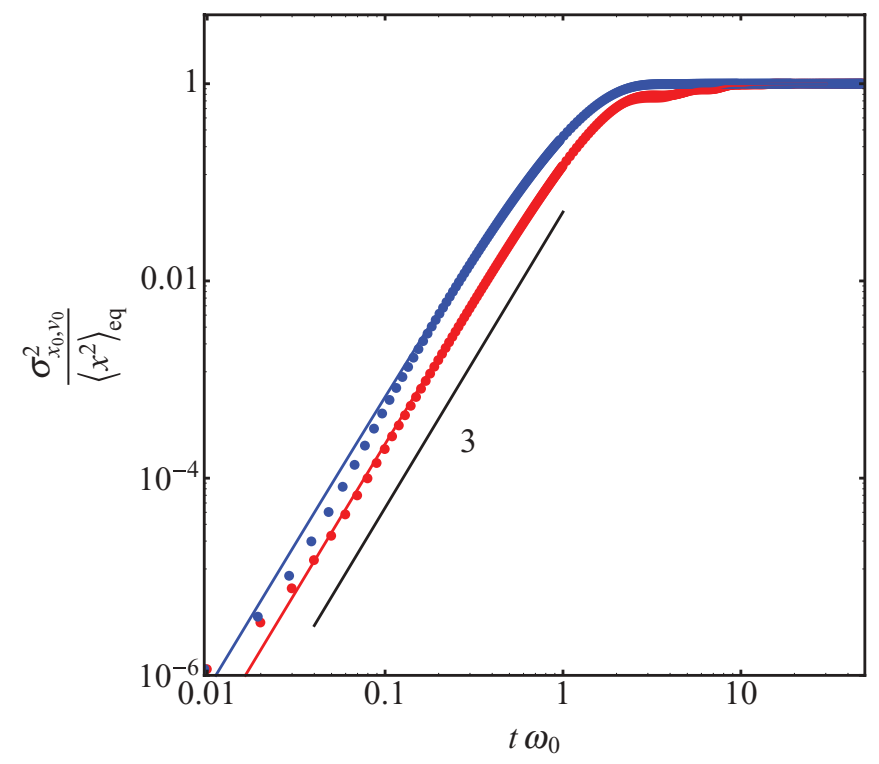

FIG. 2. (Color online) Experimentally measured particle dispersion as a function of time conditioned to the initial conditions $x_{0}$ and $v_{0}$ at $t=0$. Dispersion curves are calculated for 2500 sets of trajectories with unique initial conditions and then averaged to create the curve shown in the figure. The time is normalized by the trap frequency, $\omega_{0}$, while the dispersion is normalized by the long-time thermal particle distribution, $\left\langle x^{2}\right\rangle_{\text {eq }}$. The power law at short time (black line) is $t^{3}$, as anticipated from Eq. (6). The solid lines are the exact prediction of the Langevin model in a harmonic potential [Eq. (11)]. The red is for the low-pressure case and the blue is for the high-pressure case in the original data [13]. displacements, smaller than the bead diameter itself, but is well within the resolution of the experiment, representing the first observation of superdiffusive paths in Brownian motion.

The initial velocity $v_{0}$ determines the average trajectory, but the dispersion $\sigma_{x_{0}, v_{0}}^{2}$ describes the spreading of trajectories about that average. The comparison with Langevin prediction Eq. (11) is shown in Fig. 2, exhibiting the $t^{3}$ regime. For each subset the deterministic trajectory (i.e. for the special case where $f(t)=0$ ) of the particle is given by $\left\langle x \mid v_{0}\right\rangle$. The dispersion due to initial velocity is obtained and compared successfully to the Langevin model, as seen in Fig. 3,

$$
\frac{\sigma_{x_{0}, f}^{2}}{\left\langle x^{2}\right\rangle_{\mathrm{eq}}}=\frac{\omega_{0}^{2}}{\omega_{1}^{2}} \exp \left[-\frac{t}{\tau}\right] \sin ^{2}\left(\omega_{1} t\right),
$$

exhibiting a $t^{2}$ behavior at short times, characteristic of the "traditional" ballistic regime discussed in Ref. [13].

The discussion can be made more general by considering the case of particles starting from distributed initial positions $x_{0} \neq 0$ in the trap. The average trajectory $\left\langle x \mid x_{0}\right\rangle$ is identical to the deterministic trajectory with initial conditions $x_{0}$ and $v_{0}=\left\langle v \mid x_{0}\right\rangle$. Considering now the displacement $\delta x=x-x_{0}$, one may define also the total dispersion $\sigma^{2}$. For initial positions thermally distributed, we have

$$
\frac{\sigma^{2}}{\left\langle x^{2}\right\rangle_{\mathrm{eq}}}=2\left\{1-\exp \left(-\frac{t}{2 \tau}\right)\left[\cos \left(\omega_{1} t\right)+\frac{\sin \left(\omega_{1} t\right)}{2 \omega_{1} \tau}\right]\right\},
$$

which is now the sum of three contributions

$$
\sigma^{2}=\sigma_{x_{0}, v_{0}}^{2}+\sigma_{x_{0}, f}^{2}+\sigma_{v_{0}, f}^{2} .
$$

The two first have already been discussed above. For a fixed initial velocity $v_{0}$, and a given noise realization $f(t)$, the initial position affects the average force on the particle. The induced dispersion $\sigma_{v_{0}, f}^{2}$ is obtained (and is nearly independent of $v_{0}$ ) and is accounted for by the Langevin model as well (Fig. 3)

$$
\frac{\sigma_{v_{0}, f}^{2}}{\left\langle x^{2}\right\rangle_{\mathrm{eq}}}=\left\{1-\exp \left(-\frac{t}{2 \tau}\right)\left[\cos \left(\omega_{1} t\right)+\frac{\sin \left(\omega_{1} t\right)}{2 \omega_{1} \tau}\right]\right\}^{2},
$$

exhibiting a $t^{4}$ behavior at short times, characteristic of the existence of a constant distributed force, determined by the distributed initial position $x_{0}$, that of the trap, directing the particle's motion before it is thermalized.

The present observation of the super-diffusive regime in Brownian motion thus offers the first direct evidence of the random short-time correlated force Langevin had imagined, that force solely directing the motion of a particle initially at rest in a thermal bath. The interpretation of this new observation relies solely on the original formulation of the Langevin model with a $\delta$-correlated force, irrespective of any other effect causing possibly a correlation of the random force itself, such as hydrodynamic memory effects $[15,16]$. The presence of these effects is well-documented in the case where the fluid density is comparable to the particle density, such as in water, but is negligible in the present case of a rarefied gas. Inversely, these effects could be investigated using our method, 

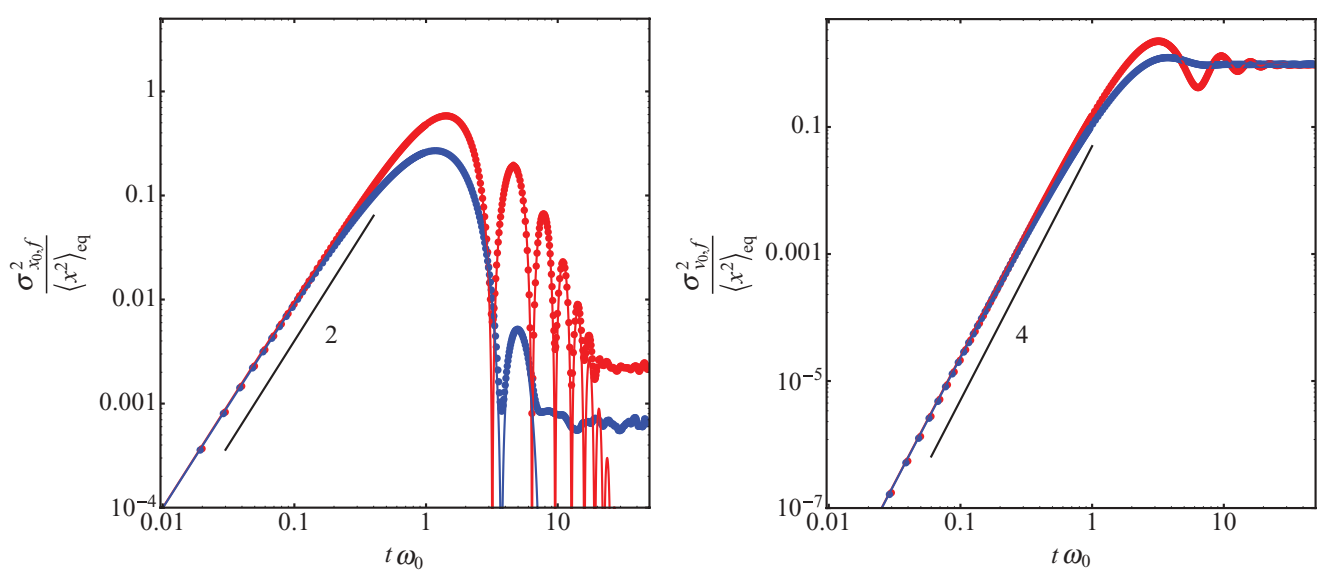

FIG. 3. (Color online) Left: Particle dispersion uniquely due to the distribution of its initial velocity $v_{0}$ : trajectories are conditioned to initial position $x_{0}$ at $t=0$ and averaged over multiple realizations of noise $f(t)$ and the thermal distribution of initial velocities. Right: Particle dispersion uniquely due to the distribution of its initial position $x_{0}$ : trajectories are conditioned to initial velocity $v_{0}$ at $t=0$, and averaged over all realization of noise $f(t)$. Dispersion curves are calculated for 50 unique initial conditions and then averaged to create the curve shown in the figure. The time is normalized by the trap frequency, $\omega_{0}$, while the dispersion is normalized by the long-time thermal particle distribution, $\left\langle x^{2}\right\rangle_{\text {eq }}$. The black line is the expected short-time power law behavior in both cases. The solid lines are the exact prediction of the Langevin model in a harmonic potential. The red curves and points correspond to the low-pressure case and the blue curves correspond to the high-pressure case [13].

which precisely singles out the force only. The study of the nature of the force acting on a particle, and of its correlation, can thus be extended to any random environment, other than Brownian motion.

In particular, we finally note the formal analogy with another $t^{3}$ super-diffusive behavior known as Richardson dispersion in high Reynolds number turbulent flows, describing the relative separation of material particles [17-20], or the coarsening of suitably prepared foams [21], an appealing analogy which may hold beyond the formal level.

E.V. thanks A. Cohen and V. Manoharan for a decisive discussion and support of the Agence Nationale de la Recherche (ANR) through Grant No. ANR-05-BLAN-0222-01. M.G.R. acknowledges support of the R.A. Welch Foundation Grant No. F-1258.
[1] A. Einstein, in Investigation on the Theory of the Brownian Movement, edited by R. Fürth (Dover Publications, New York, 1926).

[2] M. Smoluchowski, Ann. Phys. (Berlin) 21, 756 (1906).

[3] J. Fourier, Théorie analytique de la chaleur (Firmin Didot, Paris, 1822).

[4] P. Langevin, C. R. Acad. Sci. Paris 146, 530 (1908).

[5] J. Perrin, Les Atomes (Félix Alcan, Paris, 1913).

[6] G. I. Taylor, Proc. Lond. Math. Soc. 20, 196 (1921).

[7] R. Fürth, Z. Phys. 2, 244 (1920).

[8] F. Reif, Fundamentals of Statistical and Thermal Physics (McGraw Hill, New York, 1965).

[9] L. S. Ornstein, Proc. Acad. Amst. 21, 96 (1919).

[10] G. E. Uhlenbeck and L. S. Ornstein, Phys. Rev. 36, 823 (1930).

[11] S. Chandrasekhar, Rev. Mod. Phys. 15, 1 (1943).

[12] N. Wax, Selected Papers on Noise and Stochastic Processes (Dover Publications, New York, 1954).
[13] T. Li, S. Kheifets, D. Medelin, and M. G. Raizen, Science 328, 1673 (2010).

[14] R. Huang, I. Chavez, K. M. Taute, B. Lukic, S. Jeney, M. G. Raizen, and E. L. Florin, Nat. Phys. 7, 576 (2011).

[15] T. Franosch, M. Grimm, M. Belushkin, F. M. Mor, L. Forro, and S. Jeney, Nature (London) 478, 85 (2011).

[16] E. J. Hinch, J. Fluid Mech. 72, 499 (1975).

[17] L. F. Richardson, Proc. R. Soc. London A 110, 709 (1926).

[18] M. C. Jullien, J. Paret, and P. Tabeling, Phys. Rev. Lett. 82, 2872 (1999).

[19] O. Chanal, B. Chabaud, B. Castaing, and B. Hébral, Eur. Phys. J. B 17, 309 (2000).

[20] M. Bourgoin, N. T. Ouellette, H. Xu, J. Berg, and E. Bodenschatz, Science 311, 835 (2006).

[21] J. Duplat, B. Bossa, and E. Villermaux, J. Fluid Mech. 673, 147 (2011). 